\title{
Failed Systemic Methotrexate Treatment in a Rudimentary Horn Pregnancy
}

\author{
Maya Frank Wolf, MD, Talia Stahl Rosenzweig, MD, Naama Marcus Braun, MD, \\ Inbar Ben-Shachar, MD \\ Department of Obstetrics and Gynecology, Ziv Medical Center, Zfat, Israel (all authors).
}

\begin{abstract}
We report the case of a patient with unicornuate uterus and an intrarudimentary horn first-trimester ectopic pregnancy. This rare pregnancy was diagnosed by laparoscopy and did not respond to systemic methotrexate treatment. The clinical presenting symptoms and signs were similar to those of an extrauterine pregnancy. Following the failure of methotrexate treatment, a second operative laparoscopy was performed, and definitive treatment was obtained by resection of the rudimentary horn containing the pregnancy. Debates about and rationale for the elected treatment are described.
\end{abstract}

Key Words: Methotrexate, Rudimentary Horn Pregnancy, Laparoscopy.

Citation Wolf MF, Rosenzweig TS, Braun NM, Ben-Shachar I. Failed systemic methotrexate treatment in a rudimentary horn pregnancy. CRSLS e14.00112. DOI 10.4293/CRSLS.14.00112.

Copyright $\odot 2014$ SLS This is an open-access article distributed under the terms of the Creative Commons Attribution-Noncommercial-ShareAlike 3.0 Unported license, which permits unrestricted noncommercial use, distribution, and reproduction in any medium, provided the original author and source are credited.

Address correspondence to: Maya Frank Wolf, MD, Department of Obstetrics and Gynecology, Ziv Medical Center, Zfat, Israel. Telephone: +972-4-6828959; Fax +972-4-6828649; Mobile: +972-50-6262616; E-mail: homesickid@yahoo.com

\section{INTRODUCTION}

A unicornuate uterus results from unilateral failure of normal Müllerian system development. The affected Müllerian duct may not develop at all, or it may develop only partially, as either a rudimentary horn on the uterus or as a cluster of embryonic cells. This horn may not communicate with the uterus. Although most rudimentary horns are asymptomatic, others contain a functional, but not necessarily normal, endometrium that is shed cyclically. ${ }^{1}$ Although a unicornuate uterus occurs in 1 in 4020 women in the general population, ${ }^{2}$ it is more common in infertile women. Women with a unicornuate uterus had higher rates of obstetric complications, such as ectopic pregnancy (2.7\%), first-trimester abortion (24.3\%), second-trimester abortion (9.7\%), and preterm delivery (20.1\%). ${ }^{3}$ Pregnancy in a noncommunicating rudimentary horn is a well-known complication, estimated to occur in 1 in 76 000 pregnancies. ${ }^{4}$ This occurs by transperitoneal migration of sperm or fertilized ovum from the patent contralateral fallopian tube. 5 The prognosis for maternal and fetal outcome is poor, with a neonatal survival rate of $6 \%$ and uterine horn rupture risk of $50 \% .^{6}$ Most cases of rupture occur in the second trimester ${ }^{6}$ The classic treatment was to remove the uterine horn pregnancy and ipsilateral tube surgically after diagnosis by laparotomy. However, medical termination using methotrexate followed by delayed surgical resection was reported when diagnosis was obtained early in pregnancy. ${ }^{7-9}$ We describe a case of a patient with a unicornuate uterus and a noncommunicating rudimentary horn first-trimester pregnancy that did not respond to systemic methotrexate treatment and was treated with definitive surgical treatment via laparoscopy.

\section{CASE REPORT}

A 23-year-old woman was admitted to the gynecology emergency department with her sixth pregnancy due to suspected left ectopic pregnancy. Her obstetrical history revealed one left ectopic pregnancy treated by methotrexate and four early spontaneous abortions. A habitual abortion workup was performed for the patient in various other medical centers and included diagnostic hysteroscopy, revealing suspected unicornuate uterus; a threedimensional sonogram that was summarized as normal; and blood tests that excluded thrombophilia as the cause of repeated abortions. Her last menstrual period was 6 weeks before admission, and she did not use contraception. On physical examination, the patient was hemodynamically stable. The abdomen was soft and not tender. 


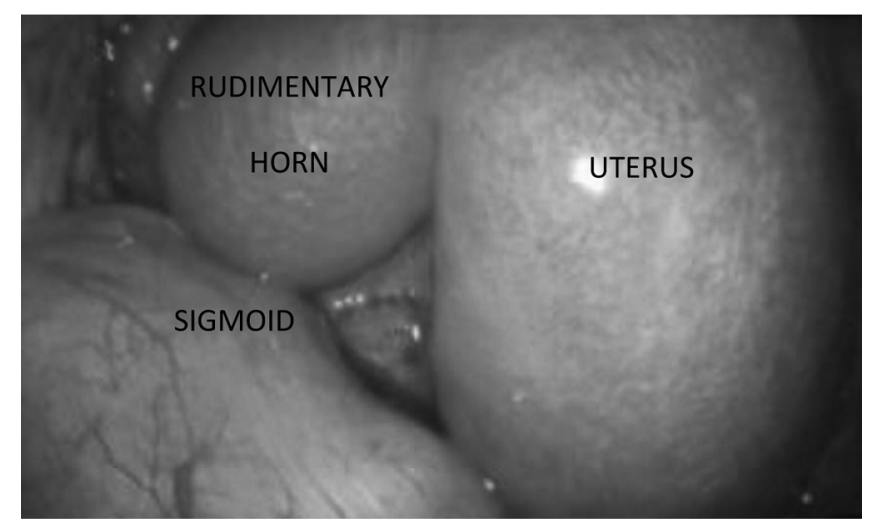

Figure 1. The unicornuate uterus and rudimentary horn via laparoscopy.

Vaginal examination was unremarkable except for mild sensitivity above the left adnexa. A transvaginal ultrasonography revealed a normal-sized retroverted uterus. An extrauterine gestational sac, containing a yolk sac and measuring $2.7 \mathrm{~cm}$, was noticed above the left ovary. The gestational sac was suspected to be attached to the uterine wall. The hemoglobin count was $11.8 \mathrm{~g} / \mathrm{dL}$, and $\beta$-hCG was 18 109. A suspected left extrauterine tubal pregnancy was diagnosed, and because of the high levels of $\beta$-hCG, the patient consented to a laparoscopic left salpingectomy or salpingostomy.

Laparoscopy revealed a unicornuate uterus and a left rudimentary horn with a normal left fallopian tube and ovary. The pregnancy was located in the left rudimentary horn (Figure 1). The right adnexa was normal. Because the patient did not consent to the resection of the uterine horn, and given the vascular nature of the rudimentary horn pregnancy, the decision was made to forego surgical treatment and instead treat the patient with systemic methotrexate. She was treated with an 8-day protocol of I.M 1 $\mathrm{mg} / \mathrm{kg}$ methotrexate alternating with folinic acid. An abdominal sonogram revealed a normal urinary system. A three-dimensional sonogram showed that the gestational sac was separate from the main uterine cavity (Figure 2).

One week after the last dose of methotrexate, the patient's $\beta$-hCG level rose to 35000 , and sonography revealed further development of the gestational sac, including an embryonic pole with pulse. The location of the rudimentary horn excluded the option of local intrarudimentary horn injection of methotrexate.

It was decided to perform a second laparoscopy to resect the rudimentary horn and ipsilateral left tube. Before the laparoscopy, a hysteroscopy was done. The right uterine

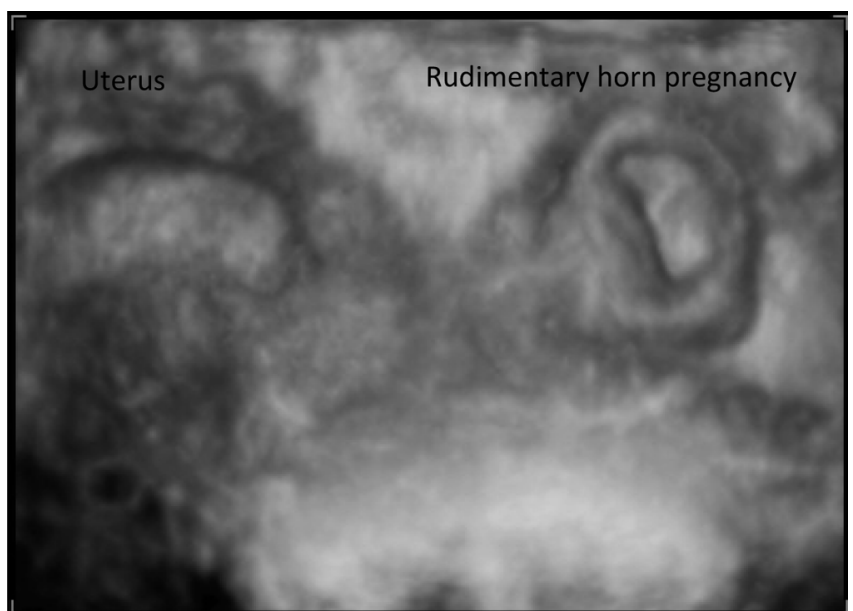

Figure 2. A three-dimensional sonogram of the gestational sac surrounded by myometrium but not connected to the uterine cavity.

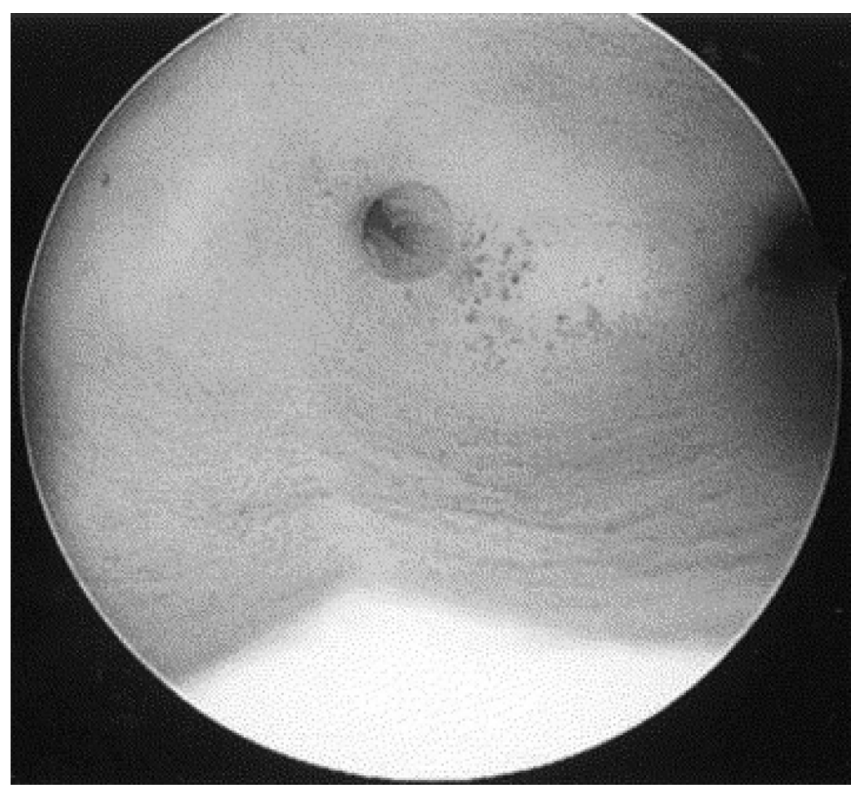

Figure 3. Hysteroscopy of a unicornuate uterus with a noncommunicating rudimentary horn.

cavity was found to be noncommunicating with the left rudimentary horn (Figure 3). Laparoscopy was started with the injection of blue dye through the cervix into the uterus. A clear passage of blue dye was observed on only the right tube, establishing the diagnosis of a left noncommunicating horn. On exploration of the pelvis, clinicians discovered that the rudimentary horn, measuring $4 \mathrm{~cm}$, was attached to the unicornuate uterus by a 20 -mm-diameter band of tissue. The left pararectal space was opened, and the iliac vessels and ureter were visualized. A left 
salpingectomy was performed. The left uterine artery was mobilized carefully and transected. Extra caution was taken to avoid damage to the left ureter. An Endoloop (Ethicon, Somerville, New Jersey, USA) was placed over the rudimentary left horn and ligated around the fibrous band before it was transected with a LigaSure (Covidien, Mansfield, Massachusetts, USA) device. The uterine horn and tube were removed from the abdomen using an Endopouch (Ethicon). Blood loss during the resection was minimal at less than $10 \mathrm{~mL}$ and did not require suction. Both operative and postoperative courses were uneventful, and a follow-up visit after 1 month was unremarkable. The pathology report revealed necrotic decidua and chorionic villi in the rudimentary uterine horn.

\section{DISCUSSION}

Rudimentary horn pregnancies are similar to tubal pregnancies sonographically in that the uterine cavity is empty and often a mass is located in the area between the uterus and the ovary. However, a rudimentary horn ectopic has a thick ring of muscle surrounding the pregnancy sac. ${ }^{10}$ This ring of muscle is due to the muscular structure of the rudimentary horn, which is histologically similar to the makeup of the uterine body. Therefore, the presence of a thick surrounding wall is indicative of an ectopic in a uterine horn, whereas its absence points to a tubal pregnancy. In addition, threedimensional sonogram may be helpful in making the diagnosis. Furthermore, the rudimentary horn endometrium is more accommodating for gestational sac development than the tube is. As a result, the extrauterine pregnancy symptoms tend to emerge later in rudimentary horn pregnancy than in tubal ectopic pregnancy.

The usual outcome of an undiagnosed rudimentary horn pregnancy is a rupture in the second trimester in about $50 \%$ to $90 \%$ of cases. ${ }^{6,11}$ Early diagnosis of this rare form of pregnancy before rupture is essential for successful management to prevent maternal morbidity and mortality. ${ }^{12}$

Scarce information exists regarding methotrexate treatment in rudimentary uterine horn pregnancy before surgical treatment.

The classic approach in rudimentary horn pregnancies is resection of the ipsilateral tube and horn via laparotomy. However, medical termination followed by delayed surgical resection is an option when diagnosis is obtained early and impending rupture seems unlikely. Antecedent medical termination may facilitate surgical resection by decreasing blood flow to the embryo. ${ }^{7} \mathrm{~A}$ few case reports have described medical treatment in a rudimentary uterine horn pregnancy. Park et $\mathrm{al}^{7}$ reported medical termination with fetal intracardiac potassium chloride and intraplacental methotrexate injections in a rudimentary horn pregnancy at 8 weeks' gestation. Magnetic resonance imaging of the pelvis was obtained to further characterize the uterine anomaly and urinary system. It revealed a rudimentary horn attached to the unicornuate uterus by a small band of tissue, and no urinary system abnormalities were detected. Laparoscopic uterine horn resection was performed 6 weeks after medical termination. Estimated blood loss was $<50 \mathrm{~mL}$, and there were no complications. Our patient was not asked to consent to intrauterine methotrexate plus potassium chloride because the initial diagnosis was a tubal pregnancy, which should have been treated easily with salpingostomy or salpingectomy, and therefore this method was unavailable to us. Edelman et $\mathrm{al}^{8}$ also reported a successful pregnancy termination of a gestation located in a noncommunicating rudimentary horn with subsequent elective laparoscopic resection.

Another case report described the nonsurgical treatment of an early rudimentary uterine horn pregnancy by local injection of methotrexate. Serial weekly $\beta$-HCG measurements were performed, and the serum $\beta$-HCG titer was measured negative on the third week. The authors concluded that local methotrexate administration can be considered an effective alternative to surgical management in the patients with no desire for future fertility. ${ }^{9}$

In our case, the rudimentary ectopic pregnancy was not affected by systemic methotrexate treatment. One cause could be the size of the gestational sac and the high levels of $\beta$-hCG. As with a cornual pregnancy, the gestational sac is implanted in the endometrium, and therefore can develop to a later gestational age. Usually, cases of rudimentary horn pregnancies are diagnosed in a later stage compared with cases of tubal pregnancy, which can be a reason for a higher failure rate of systemic methotrexate. Our patient's first pregnancy was a suspected left ectopic pregnancy at 6 weeks of gestation. Her $\beta$-hCG level was 2809. The patient was treated successfully with two methotrexate injections. It is possible that the first extrauterine pregnancy was a misdiagnosed rudimentary horn pregnancy that resolved with methotrexate treatment due to a much lower $\beta$-hCG level and a smaller gestational sac.

Local injection of methotrexate could not be performed in our case because of the location of the rudimentary horn. However, an important point is that although successful cases of local injection of methotrexate are being reported, the vascular nature of these pregnancies should be 
considered before the decision is made to use this approach.

Our patient had a hysteroscopy suggesting a unicornuate uterus before the current pregnancy. However, on admission, she reported a negative workup without providing documentation regarding the suspicious hysteroscopy. Had all of the patient's examinations and evaluations been done in a single medical center and followed by a single physician or medical team, the suspicion of a rudimentary horn could have been better evaluated. This circumstance could either have prevented the ectopic in the rudimentary horn or have led to earlier diagnosis and earlier treatment. In particular, it might have eliminated the need for an initial laparoscopy or enabled local methotrexate injections during laparoscopy.

Considering the improvements made in the past decade using an endoscopic approach in almost any gynecologic surgery, we believe that the treatment of choice for methotrexate-resistant rudimentary horn pregnancies should be laparoscopic resection of the rudimentary horn containing the pregnancy and ipsilateral tube. Because of the possibility of communicating rudimentary horn pregnancy, a diagnostic hysteroscopy should be performed before laparoscopy to exclude the option of termination of the pregnancy transvaginally.

\section{References:}

1. Jayasinghe Y, Rane A, Stalewski H, Grover S. The presentation and early diagnosis of the rudimentary uterine horn. Obstet Gynecol. 2005;105:1456-67.

2. Reichman D, Laufer MR, Robinson BK. Pregnancy outcomes in unicornuate uteri: a review. Fertil Steril. 2009;91:1886-94.

3. Reichman D, Laufer MR, Robinson BK. Pregnancy outcomes in unicornuate uteri: a review. Fertil Steril. 2009;91(5):18861894. doi:10.1016.
4. Nahum GG. Rudimentary uterine horn pregnancy. A case report on surviving twins delivered eight days apart. $J$ Reprod Med. 1997;42(8):525-532.

5. Nahum GG, Stanislaw H, McMahon C. Preventing ectopic pregnancies: how often does transperitoneal transmigration of sperm occur in effecting human pregnancy? BJOG. 2004;111(7): $706-714$.

6. Nahum GG. Rudimentary uterine horn pregnancy. The 20thcentury worldwide experience of 588 cases. J Reprod Med. 2002; 47(2):151-163.

7. Park JK, Dominguez CE. Combined medical and surgical management of rudimentary uterine horn pregnancy. JSLS. 2007; 11(1):119-122.

8. Edelman AB, Jensen JT, Lee DM, Nichols MD. Successful medical abortion of a pregnancy within a noncommunicating rudimentary uterine horn. Am J Obstet Gynecol. 2003;189(3): 886-887;PMID: 14526337.

9. Sevtap HK, Aral AM, Sertac B. An early diagnosis and successful local medical treatment of a rudimentary uterine horn pregnancy: a case report. Arch Gynecol Obstet. 2007;275(4):297-298.

10. Tsafrir A, Rojansky N, Sela HY, Gomori JM, Nadjari M. Rudimentary horn pregnancy: first-trimester prerupture sonographic diagnosis and confirmation by magnetic resonance imaging. J Ultrasound Med. 2005;24(2):219-223.

11. Sagili H, Rani R, Najundan P. Ruptured rudimentary horn pregnancy: a diagnostic dilemma and management $J$ Gynecol Surg. 2012;28(1):34-36.

12. Dahiya K, Duhan N, Nanda S. Rudimentary horn pregnancy: series of four cases and review of the literature. J Gynecol Surg. 2011;27(3):139. 